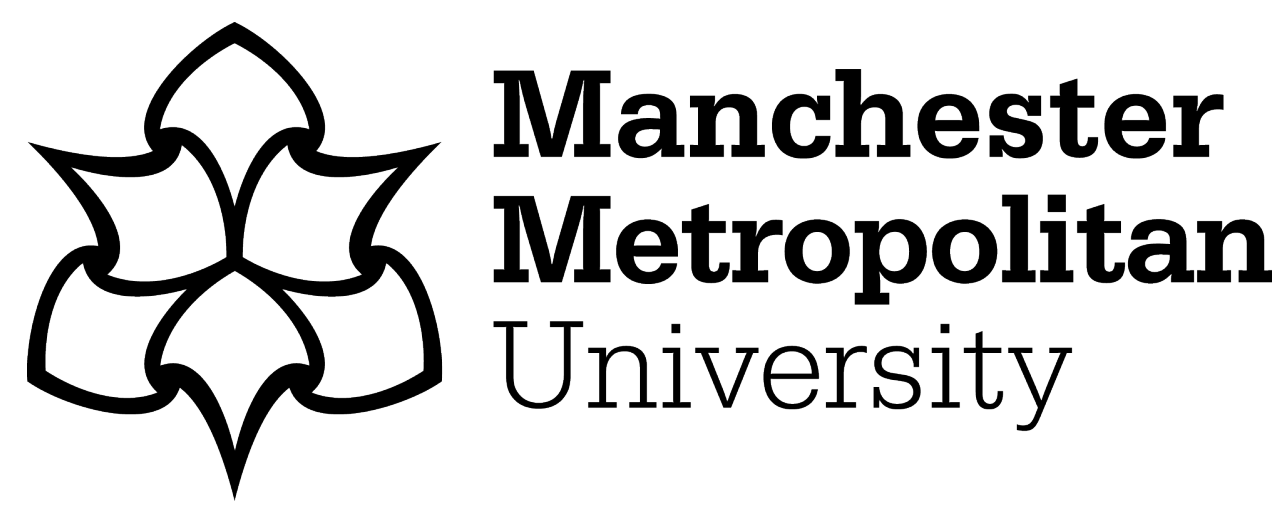

Teay, SH and Albarbar, A ORCID logoORCID: https://orcid.org/0000-00031484-8224 (2017) Adoption of MEMS technology in e-maintenance systems for rotating machinery. Insight: Non-Destructive Testing and Condition Monitoring, 59 (12). pp. 659-668. ISSN 1354-2575

Downloaded from: https://e-space.mmu.ac.uk/623538/

Version: Accepted Version

Publisher: British Institute of Non-destructive Testing

DOI: https://doi.org/10.1784/insi.2017.59.12.659

Usage rights: Creative Commons: Attribution-Noncommercial 4.0

Please cite the published version 


\title{
Adoption of MEMS technology in e-maintenance systems for rotating machinery
}

S. H. Teay, A. Albarbar

School of Engineering, Manchester Metropolitan University

All Saints Building, All Saints, Manchester, M15 6BH.

S.Teay@mmu.ac.uk

\begin{abstract}
Wireless sensor networks with on-board signal processing capabilities are becoming very attractive for machinery condition monitoring purposes. Their advantages in cost and size are becoming an important factor for deployment in a new generation of maintenance-e-maintenance. In this paper, an intelligent monitoring system for emaintenance (IMSEM) is presented using a recently developed and tested MEMS accelerometer, a low-power microprocessor and a wireless communication module. The system has a compatible framework and interface with Open Systems Architecture for Condition-Based Maintenance (OSA-CBM). Importantly, it integrates OSA-CBMdefined functions, including sensing module, signal processing, condition monitoring and health assessment; thus, the developed system can successfully reduce- the monitoring complexity and communication overhead with human operators. The performance of IMSEM is evaluated by carrying out fault diagnostic on rotating unbalance of a mechanical shaft driven by a DC motor with varying loads and speeds. By analysing the signals from the vibration signal and rotating speed, the IMSEM has the ability to carry out on-board fault detection and diagnosis. The IMSEM achieved an accuracy of $95 \%$ in detecting the fault and severity of rotor unbalance, which is based on the ISO 1940$1: 2003$.
\end{abstract}

Keywords: intelligent sensor, e-maintenance, embedded systems, MEMS, machine learning

\section{Introduction}

Maintenance strategy is recognised as one of the key factors in manufacturing and production plants for optimising their quality of productivity and efficiency. In (Kelly 2006), the total expenses in terms of maintenance costs can be greater than any investment within a complete lifecycle of a manufacturing plant, as shown in Figure 1. 
In general, there are three approaches to maintenance: reactive maintenance (fix it when it breaks), preventive maintenance (fix it when it is time) and predictive maintenance (fix it when it is right-on-time). Reactive maintenance suffers from service downtime and human risks, which degrade the overall performance of the plants. Despite preventive maintenance being effective in reducing service downtime, it is inefficient when the maintenance is made on healthy machines. One of the major overhaul facilities reports that $60 \%$ of hydraulic pumps sent in for rebuild had nothing wrong with them (Fitch 1992). In (Bloch and Hoefner 1996), they have also stated that, 'one out of three dollars spent on preventive maintenance is wasted; therefore, predictive maintenance provides optimised solution based on the aspects of cost and operating efficiency. It is a condition-based maintenance (CBM) strategy, which makes timely decisions for suitable maintenance based on the health condition and reliability of a machine.

CBM technology is well investigated and applied in many industries. Recent publications present numerous technology and software/hardware system architecture in different directions. $\mathrm{CBM}$ has also emerged in a new generation of maintenance, namely e-maintenance. It is a future vision of maintenance concept, which integrates the CBM strategy with modern technology (internet, micro-processor, smart transducer and wireless sensor network) and enterprise management. Researchers believe that, by merging e-maintenance with emanufacturing and e-business, it is a major trend in future industries, which benefits suppliers and consumers from increased equipment and process reliability ( $\mathrm{Koc}, \mathrm{Ni}$, and Lee 2002). Figure 2 highlights the integration of e-maintenance with e-manufacturing and e-business. The CBM strategy lies in the subset of e-maintenance, which serve important roles in updating production and manufacturing data with modern computer and networking system. The idea of e-business is envisioned as a centralised platform to bridge the circulation between business, manufacturing, and logistic control with optimised asset and information.

The divergence of CBM development has created a massive number of research questions and ideas. Many researchers have emphasised in developing novel techniques and analysis to tackle condition monitoring problems (He et al. 2009; Son et al. 2009; Yun et al. 2011; Ibrahim and Albarbar 2011; Li et al. 2005). To practically realise the implementation of software algorithms, other researchers have focused in developing system frameworks for specific CBM tasks (Niu, Yang, and Pecht 2010; Trutt, Sottile, and Kohler 2002; Han and Yang 2006). Moreover, wireless sensing network with intelligent algorithms have attractive potential to be deployed in CBM fields (Son et al. 2009; Wang and Jianu 2010). Hence, it is necessary to take on the task of integrating a wide variety of software and hardware technology into a mutual 
platform for these components. Proper standards or an emergence of dominant design is required in order to direct the development and cultivate the benefit of both customers and developers. Open system architecture for condition-based maintenance (OSA-CBM) is an open standard to facilitate the technological growth of CBM system. The system framework and architecture of OSA-CBM is well discussed in official documents (Penn State University, The Boeing Company, and MIMOSA 2006). It is becoming more popular in CBM, and its standardisation issues are well discussed in (Lee, Gao, and Schneeman 2002) and (Bengtsson 2003).

Due to there being limited flexibility in integrating the CBM system designs from different vendors and developers, OSA-CBM outlines a standardised system framework, aiming to:

- improve the communication paradigm between the sensor platform with other maintenance modules;

- develop and identify research questions in current CBM technology;

- increase competency and rapid technology development; and

- reduce costs.

OSA-CBM can be divided into seven functional layers, as illustrated in Figure 3 (Niu, Yang, and Pecht 2010). Each layer has the capability of requesting data from any functional layer, as needed. Data is usually transmitted between adjacent functional layers; however, it is difficult to transfer data between platforms due to the different formats and information management of different vendors and manufacturers. In an effort to provide a unified interface for data transmission, OSA-CBM specifies how the data should circulate within the system using the eXtensible Mark-up Language (XML). By using the XML schema provided by OSA-CBM, all functional modules compliant with the OSA-CBM standard can easily exchange data within the monitoring system.

The implementation of the intelligent sensor is the current trend in machinery condition monitoring. Benefitting from the significant growth of microelectronic technology, it is possible to design an intelligent monitoring system for e-maintenance (IMSEM), where its structure is compatible with OSA-CBM, thus providing higher interoperability and adaptability in future deployment. Hence, it reduces redundant implementation requirements by simplifying the information circulation within the whole OSA-CBM architecture, as shown in Figure 4. The 
modular structure of IMSEM has advantages in terms of minimising the size and complexity of internal communication, thus reducing communication workload.

In an effort to investigate the deployment of IMSEM on CBM system, three main components are outlined, namely the sensing node, computational unit and interface terminal. Their current developments are discussed as listed below:

- Sensing node: Various sensors are available as suitable sensing nodes for IMSEM. Wang et al. presented an embedded sensing unit using integrated circuit piezoelectric accelerometers (Wang and Jianu 2010); however, conventional accelerometers have limited functionality and incur high costs. The recent growth of MEMS is an important technology in developing IMSEM. Their characteristics and applications in CBM have been well discussed in (Alhussein Albarbar et al. 2008; A. Albarbar et al. 2009). Moreover, its durability and ability to survive at high shock levels and temperatures in harsh environment have also been proven (Stauffer, Dutoit, and Arbab 2006). In this paper, MEMS accelerometer is successfully implemented with an on-board processor to carry out fault diagnostic.

- Computational unit for data acquisition, signal processing and fault diagnostics: The embedded system plays important roles in developing a decentralised IMSEM system. The applicability of the embedded microprocessor is solely dependent on its application in CBM. In this paper, a periodic monitoring system (also known as an offline condition monitoring system) is selected. The machinery signals are measured at selected time intervals in the field, with data acquisition and analysis then carried out for fault diagnosis and trend analysis. This has advantages in terms of saving limited resources and power consumption of IMSEM. Furthermore, it could carry out better on-board processing algorithms, which take longer computation time.

- Communication terminal: Wireless modules, such as the XBee (Digi International Inc. 2014), are a promising communication device in terms of establishing a wireless sensor network. It has great benefits in terms of reducing wiring costs, which is one of the critical cost components in a CBM installation. Normal the XBee configurations communicate in ASCII text representations; therefore, it has a compatible interface module with OSA-CBM. Relevant monitoring information could be sent in XML format. 
By selecting suitable technology, this paper aims to design an embedded IMSEM with the objective to establish wireless sensor network (WSN) for CBM system. The IMSEM has complete features to carry out signal acquisition, signal conditioning, feature extraction and fault detection. By accumulating vibrating signal and rotating speed, it has the ability to extract relevant feature components, indicating the fault level of rotor unbalance of a DC motor. A simple data fusion layer is also implemented on the IMSEM to conclude condition relevant information. The paper is organised as follows: in the next section, a proposed system architecture of IMSEM is presented. In Section 3, the computational level of IMSEM is presented; this includes the resource management of IMSEM and data fusion level. In Section 4 , a working prototype is deployed in a DC motor test rig for practical testing. The results and discussion of the experiment are elaborated in Section 5. Finally, in Section 6, some conclusions are drawn and future works suggested.

\section{Proposed Intelligent Monitoring System for E-Maintenance}

The proposed IMSEM comprises two sensing nodes (MEMS accelerometer and a Hall effect sensor), two microcontroller units (MCU), a removable data storage unit and an XBee as the wireless communication module. These have extended the capability of the IMSEM. Furthermore, five functional blocks of OSA-CBM (sensing channel, data-acquisition, signal processing, condition monitoring and health assessment) can be carried within the IMSEM, thus reducing the communication overhead with other system platforms. The system architecture of IMSEM is shown in Figure 5. In order to evaluate the performance of the proposed system, a working prototype is developed, as shown in Figure 6. All the hardware components are built in modular designs, and their functionalities and capabilities can be independently tested.

\subsection{Sensing Channel}

For the sensing channel (signal acquisition module), the MEMS accelerometer and digital Hall effect sensor are used to measure the vibration and angular speed of the rotating machinery, respectively. These sensors have advantages of incurring low cost, facilitating easy installation, and having lesser power factors compared with other sensing modules, such as conventional piezoelectric accelerometer and digital encoder. Here, a newly designed and tested MEMS accelerometer is selected as a sensing element for proposed IMSEM [2]. It has a good range of 
effective frequency band (up to $22 \mathrm{kHz}$ ), and works at $3.3 \mathrm{~V}$ operation. It also has low power consumption and low noise (typical $2.5 \mathrm{~mA}$ and $4 \mathrm{mg} / \sqrt{\mathrm{Hz}}$ ).

A Hall effect sensor is combined with additional passive electronic circuitry to give digital output. Similar to the ALDXL001-70, the Hall effect sensor is able to work at 3.3V operation.

The signal from the MEMS and Hall effect sensor are measured through a peripheral mixed signal MCU. This peripheral mixed signal MCU has a proprietary M8C core, which serves as a programmable anti-aliasing filter to prevent the aliasing effect. With an in-built 12bit ADC module, the IMSEM is able to accumulate vibration signals (analogue) and rotating (digital) signals simultaneously at a maximum sampling frequency of $5 \mathrm{kHz}$.

\subsection{Anti-aliasing Filter}

To satisfy sampling theorem, the frequency components of sampled signal must not exceed one-half of the sampling frequency. A peripheral mixed signal microcontroller with programmable continuous low-pass filter is used in designing IMSEM. It has many advantages over conventional electronic circuitry, such as in terms of interface, size and cost. The programmable filter uses switched capacitor technology to alternate the impedance of the circuitry. Its respective circuitry can be illustrated as shown in Figure 7. It works by continuously moving electrical charges in and out of capacitors by toggling the switches with respective switching frequency, $f_{c l k}$. The relationship of voltage, switching frequency and resistance is expressed in (1).

$$
\frac{V_{2}-V_{1}}{I}=\frac{1}{f_{c l k}}=R
$$

With built-in integrated switched capacitor module and operational amplifier, the peripheral mixed signal MCU is utilised as an analogue anti-aliasing filter, which, with its cut-off frequency, is adjustable to facilitate different monitoring applications. The switched capacitor module plays an important role in generating variable resistance by continuously toggling the switches with respective clock frequency, $f_{c l k}$. A two-pole low-pass filter is used as programmable anti-aliasing filter, as shown in Figure $8 . \emptyset_{1}$ and $\emptyset_{2}$ are switching frequencies which invert with one another. The addition of $\emptyset_{1}$ and $\emptyset_{2}$ is equivalent to $f_{c l k}$, presenting a complete switching cycle. $C_{A}, C_{B}, C_{1}, C_{2}, C_{3}, C_{4}$ which are respective capacitance values for each capacitor. Stated in (Cypress Semiconductor Corporation 2011), the transfer function of the low-pass filter can be expressed in (2). 


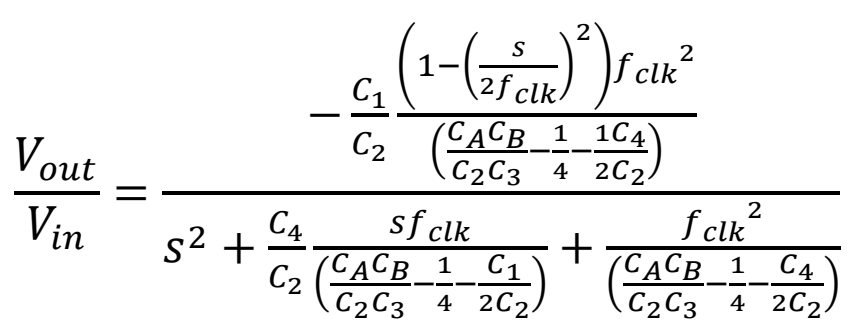

where its cut-off angular velocity can be derived as shown:

$$
\omega_{c}=\frac{f_{c l k} \sqrt{C_{2} C_{3}}}{\sqrt{\left(C_{A} C_{B}-\frac{C_{2} C_{3}}{4}-\frac{C_{4} C_{3}}{2}\right)}}
$$

In this application, the default sampling frequency is $5 \mathrm{kHz}$. The cut-off frequency of $2.5 \mathrm{kHz}$ is pre-defined in the peripheral mixed signal CPU. The anti-aliasing filter is - evaluated, as shown in Figure 9.

\subsection{Signal Processing and Feature Extraction Module}

Signal processing and feature extraction are carried out at the core computational unit of IMSEM. The core computational unit has a $65 \mathrm{kBytes}$ memory space reserved for data acquisition. With its internal data buffer, the IMSEM is able to carry out continuous data logging with any sampling frequency below $5 \mathrm{kHz}$. In higher sampling frequency configuration, the IMSEM has a maximum burst period sampling period of 19 seconds at $10 \mathrm{kHz}$, which is sufficient to accumulate useful measurements. For power spectrum analysis, the IMSEM has the ability to carry out 2048-points FFT algorithms with the resolution of $2.4414 \mathrm{~Hz} / \mathrm{Bin}$ in $5 \mathrm{kHz}$ sampling frequency and $4.8848 \mathrm{~Hz} / \mathrm{Bin}$ in $10 \mathrm{kHz}$ sampling frequency respectively. In order to avoid losing precision information, the quantised signals are stored in either 16-bit or singlefloating point format. Such criteria have ensured that accurate numerical information can be computed within the IMSEM architecture. To evaluate the machinery health with statistical feature components, a data fusion layer is also implemented in a core computational unit. This will be discussed further in Section 3.

Five feature components are extracted from the time-domain and frequency domain, as detailed in Table 1.

Table 1: Feature components extracted from core computational unit 


\begin{tabular}{cccc}
\hline Feature component & Source & Domain & Formula \\
Mean average & Vibration & Time & $\bar{x}=\frac{1}{k} \sum_{i=1}^{k} x_{i}$ \\
RMS & Vibration & Time & $x_{\text {rms }}=\sqrt{\frac{1}{k} \sum_{i=1}^{k} x_{i}^{2}}$ \\
Standard deviation & Vibration & Time & $x_{\sigma}=\sqrt{\frac{1}{k-1} \sum_{i=1}^{k}\left(x_{i}-\bar{x}\right)^{2}}$ \\
Kurtosis & Vibration & Time & $x_{k u r}=\frac{1}{k} \sum_{i=1}^{k}\left(\frac{x_{i}-\bar{x}}{x_{\sigma}}\right)^{4}$ \\
Peak & Vibration & Frequency & $x_{\text {max }}=\max (F F T(X))$ \\
\hline
\end{tabular}

where $x$ is the input signal. $k$ and the number of samples of $x$.

\subsection{Removable Data Storage Module Compliant with OSA-CBM Format}

Due to limited memory, a removable data storage module is introduced to record user configurations, raw data signals, extracted feature components and monitoring result. The implementation of removable data storage enables a larger non-volatile storage, extending the capability of IMSEM in the long-term monitoring process. All information in the data storage module is saved in ASCII text representations, in which the information can be easily retrieved or edited on an end-user interface. To build an OSA-CBM-compatible system, a compliant $\mathrm{XML}$ schema is predefined in a removable mass storage device. The authors used a commercial available secure digital (SD) card as a removable mass storage device as it provides direct access to normal computers with appropriate file system architecture: File allocation table (FAT) or the new technology file system (NTFS). With huge memory capacity of a SD card interfacing with the data storage module, the IMSEM can save five outputs in ASCII text formats: vibration signal, rotating speed, feature components, real frequency spectrums and sensor configurations. Their respective XML schemas based on the OSA-CBM standards are tabulated in Table 2.

Table 2: XML document type for IMSEM output respective to their XML schema

\begin{tabular}{cccc}
\hline Output & XML Schema & Root element & XML filename \\
\hline Vibration signal & DataAcquisition.xsd & DAWaveform & DAVibration.xml \\
Rotating speed & DataAcquisition.xsd & DAWaveform & DASpeed.xml \\
Frequency spectrum & DataManipulation.xsd & RealFrqSpect & DMfft.xml
\end{tabular}




\begin{tabular}{cccc} 
Feature components & DataManipulation.xsd & DMDataSeq & DMData.xml \\
Configurations & Configuration.xsd & Configuration & ISConfig.xml \\
\hline
\end{tabular}

Vibration and rotating speeds are retrieved from the accelerometer and Hall effect sensor, in which the information is extracted from data acquisition module. The frequency spectrum and computed feature components are the processed data from the data manipulation module. In order to avoid confusion, the OSA-CBM outlines namespaces for different functional layers. For example, 'DAWaveform' under 'DataAcquisition.xsd' is used for waveform information from data acquisition layer, whilst 'DMDataSeq' under 'DataManipulation' is used for saving the sequence of data information in the data manipulation layer. With pre-programmed algorithms, the IMSEM has the ability to arrange information in OSA-CBM based XML documents, ready for future implementation with other devices.

\subsection{Workflow of IMSEM}

Figure 10 depicts the data flow within the IMSEM. Firstly, the start-up process takes place by loading initial settings from an external memory drive into a core computational unit. The wireless module, core computational unit and data storage module will then be in its ready mode. In this condition, the IMSEM waits for commands from users or other platforms. The IMSEM will proceed to the next phase if there is no command for a period of time interval. At this stage, the IMSEM enters the busy mode (interrupt service handler from wireless module is disabled) for condition monitoring. Upon completing the monitoring tasks, the IMESEM returns to ready mode, pending further user command, or continues to the next time interval.

\section{Data Fusion Layer using SVM}

The IMSEM has the ability to extract five feature components, mapping them into multidimensional space. These feature components need to be assessed in the data fusion layers for classification purpose. The Support vector machine (SVM), a supervised learning model, is integrated into the data fusion layer of the IMSEM. The SVM was originally formulated by Vladimir N. Vapnik in (Cortes and Vapnik 1995). Since then, the SVM has drawn attention from various research fieldsand it is widely implemented as a supervised learning classifier for machinery condition monitoring. Qiang Miao et al. have compared SVM classification characteristics with the Markov Model in gearbox fault detection (Miao, Huang, and Fan 2007). By giving a set of training examples with classified categories, SVM builds a generalisation 
model that assigns new examples into a suitable category. Figure 11 shows an example of mapped samples on a two-dimensional feature space. The mapped data points are represented in squares and circles, which are respectively classified as negative and positive. The main idea of the SVM is to establish the separating region as classification boundary between the two different classes. Ideally, the hyperplane of the boundary is placed at the maximum distance of two nearest supporting data points.

With the given number $n$ of feature component, the input $x$ can be expressed in $x_{i},(i=$ $1,2, \ldots N)$. Two output classes are taken into account, denoted as $y_{i} \in\{-1,+1\}$. The formula of hyperplane, separating data, can be expressed as follows:

$$
f(x)=w^{T} x+b=\sum_{i=1}^{N} w_{i} x_{i}+b=0
$$

where $w$ is $\mathrm{N}$-dimensional vector and $b$ is a threshold to separate hyperplane. The same function can be used to classify new input data points, as based on the trained generalisation model $f(x)$, given by:

$$
\hat{y}=\operatorname{sgn}\left(w^{T} \cdot x+b\right)
$$

The objective of SVM is to determine the suitable $w$ vector. Considering the slack variables $\epsilon_{i}$ the optimal hyperplane separating the data can be obtained by reducing the cost function, as shown:

$$
\min _{w, b, \epsilon}\left(\frac{1}{2} w^{T} w+C \sum_{i=1}^{N} \epsilon_{i}\right)
$$

subject to

$$
\begin{aligned}
& y_{i}\left(w^{T} x_{i}+b\right) \geq \rho-\epsilon_{i} \\
& \epsilon_{i} \geq 0, \quad \forall_{i}=1, \ldots N
\end{aligned}
$$

where $C$ is the error penalty. The calculation can be simplified by transforming the problem with the Kuhn-Tucker condition into an equivalent space of Lagrangian variables $\alpha=$ $\left[\alpha_{i}, \ldots, \alpha_{n}\right]^{T}$, yielding: 


$$
\min _{w, b, \epsilon} \frac{1}{2} w^{T} w-\sum_{i=1}^{N} \alpha_{i} y_{i}\left(w \cdot x_{i}+b\right)+\sum_{i=1}^{N} \alpha_{i}
$$

Hence, by solving the dual optimisation problem, the coefficient $\alpha_{i}$ can be determined, thus leading to solving $w$ in equation (6). The classification process is based on the generalised parameters $\alpha_{i}$. Equation (5) can be rearranged with $w=\sum \alpha_{i} y_{i} x_{i}$, yields,

$$
\hat{y}=\operatorname{sgn}\left(\sum_{i=1}^{N} \alpha_{i} y_{i} x_{i}+b\right)
$$

In equation (9), there is only 1 feature input taken into account. In the data fusion layer of the IMSEM, multiple feature components are required as feature vectors for the classification function. The multi-dimensional feature vectors can be projected into a higher dimensional feature space using a kernel function $K\left(x_{i}, x_{j}\right)$, where $j$ defines the number of feature components. The kernel function $K\left(x_{i}, x_{j}\right)$ is a useful technique which returns a dot product of the high dimensional feature space of original input data points. By substituting the kernel function into equation (9), equation (10) is the result. There are several kernel functions that are common in SVM classification. To test the validity of SVM, a linear kernel function, $K\left(x_{i}, x_{j}\right)=\left(x_{i}^{T} \cdot x_{j}\right)$ is used and integrated into the IMSEM.

$$
\hat{y}=\operatorname{sgn}\left(\sum_{i, j=1}^{N} \alpha_{i} y_{i} K\left(x_{i}, x_{j}\right)+b\right)
$$

With linear kernel:

$$
\hat{y}=\operatorname{sgn}\left(\sum_{i, j=1}^{N} \alpha_{i} y_{i}\left(x_{i}^{T} \cdot x_{j}\right)+b\right)
$$

There are various types of source code available to apply the SVM classification. An open source library for support vector machines (LIBSVM) is chosen as the main source code for developing the SVM algorithms for the IMSEM. Written by Lin et al. and published in (Chang and Lin 2011), this provides an efficient library for the SVM classification and regression. The implementation of the LIBSVM on embedded systems is proven in (Boni, Pianegiani, and Petri 
2007). A Linear kernel is chosen as a testing kernel function for the experiment. The performance and characteristic of SVM with extracted feature components are discussed in Section 5 .

\section{Condition monitoring on a DC motor test rig}

An experimental DC motor test rig is used to simulate rotating unbalance. The disc-shaped rotor is mounted between bearings. A single plane balancing is considered as the rotor thickness is smaller than its radius. The diameter of the rotor is $0.072 \mathrm{~m}$, weighing $0.606 \mathrm{~kg}$. These properties are important in terms of establishing the permissible residual unbalance using ISO 1940-1:2003 ${ }^{1}$. In ISO 1940-1:2003, the balance quality grades for various machines are outlined, known as $G$. The grade $G$ is a product term of specific unbalance, $e$ and the angular velocity at maximum speed, $\omega$ :

$$
G=e \times \omega
$$

In practice, the grade $G$ could be defined based on the guidelines provided by ISO 19401:2003, as tabulated in Table 3. The permissible residual unbalance, $U_{p}$ (measured in $\mathrm{g} \cdot \mathrm{mm}$ ), on the other hand, can be computed using:

$$
U_{p}=9549 \times G \frac{m_{r}}{W}
$$

where $m_{r}$ is the rotor mass in $\mathrm{kg}$ and $W$ is the maximum rotating speed in rpm. The ISO 1940-1:2003 standard can be illustrated as shown in Figure 12.

Table 3: Extracted guidance for balance quality grades for rotors in a constant state (ISO 1940-1 Mechanical Vibration - Balance Quality Requirements for Rotors in a Constant (rigid) State Part 1: Specification and Verification of Balance Tolerances, n.d.)

\begin{tabular}{cc}
\hline Machinery types: General example & Balance quality grade, $\mathrm{G}$ \\
\hline $\begin{array}{c}\text { Crankshaft drives for large slow marine diesel engine (piston speed } \\
\text { below 9m/s), inherently unbalanced }\end{array}$ & 4000 \\
$\begin{array}{c}\text { Crankshaft drives for large slow marine diesel engines (piston speed } \\
\text { below 9m/s), inherently balanced }\end{array}$ & 1600 \\
Crankshaft drives, inherently unbalanced, elastically mounted & 630 \\
Crankshaft drives, inherently unbalanced, rigidly mounted & 250
\end{tabular}

\footnotetext{
${ }^{1}$ International standard 1940-1:2003 Mechanical vibration - Balance quality requirements for rotors in a constant (rigid) state. This standard has been reviewed and confirmed in 2013. [Online] Available: www.iso.org
} 
Agricultural machinery, crankshaft drives, crushing machines, drive shafts (cardan shafts, propeller shafts)

Electric motors and generators (of at least $80 \mathrm{~mm}$ shaft height), of maximum rated speed up to $950 \mathrm{rpm}$

Electric motors and generators (of at least $80 \mathrm{~mm}$ shaft height), of maximum rated speed above $950 \mathrm{rpm}$

The experimental DC motor test rig has a rated rotating speed of up to 2000rpm. The balance quality grade of 2.5 is used to determine the permissible residual unbalance of the motor testbed. The trial weight is loaded on the axial plane of the rotating disc. In the balancing theory, the unbalance coefficient, $U_{p}$ can be determined by establishing the trial mass, $m_{t}$ and distance from unbalance to the centre of rotation axis, $d: U=m_{t} \times d$. This serves as a benchmark to detect the faulty condition of a DC motor test rig. The threshold for training data is identified as based on the calculated $U_{p}$.

The DC motor generates several ranges of rotating speed, tending to carry out fault diagnosis on rotor unbalance under variable rotating speeds. The MEMS accelerometer is placed at the bearing housing, whereas the Hall effect sensor is placed at the side of the rotating disc, as shown in Figure 13. Moreover, 63 sets of data are taken with trial weights under the rotating speed. The first 25 sets are used to train the data fusion model, whilst the other 38 sets are used for testing. Currently, the IMSEM has a binary classification output from the data fusion layer: \{healthy, alarm\}. The data is saved under an XML format or can be transmitted in a simplified format with user commands. The performance and generated monitoring result is discussed in Section 5 .

\section{Results and Discussion}

The alarm threshold level is firstly specified according to ISO 1940-1:2003 standard. Figure 14 shows the threshold level respective to different rotating speeds. In this experiment, the rotating disc is applied with different trial weights: $0.5 \mathrm{~g}, 0.9 \mathrm{~g}, 1.2 \mathrm{~g}, 1.5 \mathrm{~g}, 1.8 \mathrm{~g}$ and $4.5 \mathrm{~g}$. The rotor unbalance and its residual permissible threshold are computed using equations (12) and (13). The slotted line classifies the threshold region: The region above the slotted line presents the residual unbalance coefficients while the region below the slotted line presents the healthy region. The dotted horizontal lines present the machinery condition with different loads and 
speeds. It should be noted that, the dotted lines of $0.5 \mathrm{~g}, 0.9 \mathrm{~g}, 1.2 \mathrm{~g}, 1.5 \mathrm{~g}$ and $1.8 \mathrm{~g}$ and $4.5 \mathrm{~g}$ falls into the healthy region when the rotational speed is low. This clearly satisfies the statement in ISO 1940-1:2003: Lower rotational machines have higher permissible residual unbalance.

A total of 63 sets of vibration and Hall effect signals are acquired for training and testing purpose (11 sets of healthy data and 52 sets of faulty data). Amongst these, 25 sets of data are used for generating the SVM classification model. All datasets have their respective rotor unbalance calculated for training and evaluation purpose.

The vibration signals of acquired datasets are shown in Figure 15. These vibration signals are then transformed to frequency domain using a 2048-point FFT. It should be noted that the power spectrum peak increases in higher load and speed. The digital signals from Hall effect sensor are acquired for accurate rotating speed detection. The Hall effect sensor is configured in an active-high mode, giving a low signal pulse when a magnetic object is approaching the sensing terminal. In this experiment, 1 digital pulse is given out in one rotating cycle. Hence, the rotating speed can be accurately acquired by multiplying the digital signal period with the sampling frequency of IMSEM, $f_{s}$.

With an in-built LIBSVM, the optimisation computation is completed in 17 iterations. After the experiment, the IMESEM reached an accuracy of $94.74 \%$, where 36 out of 38 data sets are predicted correctly. This performance is compared with the SVM classification with RBF kernel using a computer. Both of them have the same accuracy of $94.74 \%$, as tabulated in Table 4. The training and test samples are mapped onto the ISO1940-1 guidelines, as shown in Figure 16. The accuracy error is expected from the classification over-fitting due to the stochastic characteristics of mean and kurtosis components. In Figure 17, it is obvious that rms, standard deviation and peak have greater impact than mean and kurtosis statistically. These 3 feature components provide a relatively linear response with respect to the increased unbalance and speed. By removing the mean and kurtosis from the feature component list, the IMSEM reached an accuracy of $100 \%$.

Table 4: The comparison of kernel type for SVM classification

\begin{tabular}{ccc}
\hline Type of kernel & Linear (IMSEM) & RBF (Computer) \\
\hline Iteration of optimisation & 17 & 15 \\
Correct fault detection & 36 out of 38 & 36 out of 38 \\
Accuracy & $94.74 \%$ & $94.74 \%$ \\
\hline
\end{tabular}




\section{Conclusion}

This paper has presented a system framework of the IMSEM, which is possible to deploy in a CBM-based operating plant. The IMSEM has the ability to acquire, extract and generate a CBM report to higher level devices or human interface. The format of the information report is generated in XML, which is compliant with all the OSA-CBM compatible system. With an additional memory capacity (provided by removable flash drive), the IMSEM calculates statistical parameters and frequency domain analysis, which is an advantageous over currently available devices. A supervised learning algorithm based on the SVM is implemented on data fusion layer to generate condition monitoring results. However, several issues are being taken into account in order to strengthen the applicability of IMSEM in CBM fields:

- Power management: the IMSEM has no intelligence in managing power consumption. Currently, the IMSEM consumes an average of $1.05 \mathrm{~W}(0.21 \mathrm{~A}$ at $5 \mathrm{~V}$ operation) at full operation and idle listening mode. The power management strategy should be carried out at the hardware and software levels to prolong the operating life of the IMSEM on a CBM system.

- Data fusion: the data fusion layer involves a linear kernel of SVM classification. It is possible to implement a higher level of system kernel to improve generalisation capability. Moreover, coding optimisation needs to be carried out so as to minimise the resource consumption of data fusion layer in the whole architecture of the IMSEM.

Finally, the developed IMSEM sends XML data in ASCII format, which involves massive communication workload. To minimise the communication overhead, it is possible to send a compliant information structure to the target device, as specified in an Open Standard Architecture-Enterprise Application Integration (OSA-EAI). This will be further investigated in the coming research.

\section{References}

Albarbar, A., A. Badri, Jyoti K. Sinha, and A. Starr. 2009. "Performance Evaluation of MEMS Accelerometers." Measurement 42 (5): 790-95. doi:10.1016/j.measurement.2008.12.002. Albarbar, Alhussein, Samir Mekid, Andrew Starr, and Robert Pietruszkiewicz. 2008. "Suitability of MEMS Accelerometers for Condition Monitoring: An Experimental Study.” Sensors 8 (2): 784-99. doi:10.3390/s8020784.

Bengtsson, Marcus. 2003. "Standardization Issues in Condition Based Maintenance." In . http://mdh.diva-portal.org/smash/record.jsf?pid=diva2:301566.

Bloch, Heinz P., and John J. Hoefner. 1996. Reciprocating Compressors: Operation \& Maintenance. Gulf Professional Publishing. 
Boni, A., F. Pianegiani, and D. Petri. 2007. "Low-Power and Low-Cost Implementation of SVMs for Smart Sensors." IEEE Transactions on Instrumentation and Measurement 56 (1): 39-44. doi:10.1109/TIM.2006.887319.

Chang, Chih-Chung, and Chih-Jen Lin. 2011. "LIBSVM: A Library for Support Vector Machines." ACM Transactions on Intelligent Systems and Technology 2 (3): 27:1-27:27.

Cortes, Corinna, and Vladimir Vapnik. 1995. "Support-Vector Networks." Machine Learning 20 (3): 273-97. doi:10.1023/A:1022627411411.

Cypress Semiconductor Corporation. 2011. CY8C29466, CY8C29566, CY8C29666, CY8C29866. 3812013 Rev. *S. Cypress Semiconductor Corporation.

Digi International Inc. 2014. XBee/XBee-PRO RF Modules. 90000982_P. ftp1.digi.com/support/documentation/90000982_P.pdf.

Fitch, Jim. 1992. "Proactive Maintenance Can Yield More than a 10-Fold Savings Over Conventional Predictive/Preventive Maintenance Programs." In Predictive Maintenance Indaba Proceedings, 1-9. South Africa: Noria Corporation. http://www.oilfiltrationsystems.com/media/pdfs/case_studies/proactive.pdf.

Han, Tian, and Bo-Suk Yang. 2006. "Development of an E-Maintenance System Integrating Advanced Techniques." Computers in Industry 57 (6): 569-80. doi:10.1016/j.compind.2006.02.009.

He, Qingbo, Ruqiang Yan, Fanrang Kong, and Ruxu Du. 2009. "Machine Condition Monitoring Using Principal Component Representations." Mechanical Systems and Signal Processing 23 (2): 446-66. doi:10.1016/j.ymssp.2008.03.010.

Ibrahim, G R, and A Albarbar. 2011. "Comparison between Wigner-Ville Distribution- and Empirical Mode Decomposition Vibration-Based Techniques for Helical Gearbox Monitoring." Proceedings of the Institution of Mechanical Engineers, Part C: Journal of Mechanical Engineering Science, June. doi:10.1177/0954406211403571.

ISO 1940-1 Mechanical Vibration - Balance Quality Requirements for Rotors in a Constant (rigid) State Part 1: Specification and Verification of Balance Tolerances. n.d. ISO 1940-1:2003(E). International Standard.

Kelly, Anthony. 2006. Strategic Maintenance Planning. Oxford: Elsevier Butterworth-Heinemann. http://capitadiscovery.co.uk/mmu/items/1748816.

Koc, M., J. Ni, and J. Lee. 2002. "Introduction of E-Manufacturing." In Proceedings of the 5th International Conference on Frontiers of Design and Manufacturing (ICFDM'2002), 43-47.

Lee, K., R.X. Gao, and R. Schneeman. 2002. "Sensor Network and Information Interoperability Integrating IEEE 1451 with MIMOSA and OSA-CBM." In Proceedings of the 19th IEEE Instrumentation and Measurement Technology Conference, 2002. IMTC/2002, 2:1301-5 vol.2. doi:10.1109/IMTC.2002.1007145.

Li, Yuhua, Fengshou Gu, Georgina Harris, Andrew Ball, Nick Bennett, and Ken Travis. 2005. "The Measurement of Instantaneous Angular Speed.” Mechanical Systems and Signal Processing 19 (4): 786-805. doi:10.1016/j.ymssp.2004.04.003.

Miao, Qiang, Hong-Zhong Huang, and Xianfeng Fan. 2007. "A Comparison Study of Support Vector Machines and Hidden Markov Models in Machinery Condition Monitoring." Journal of Mechanical Science and Technology 21 (4): 607-15. doi:10.1007/BF03026965.

Niu, Gang, Bo-Suk Yang, and Michael Pecht. 2010. "Development of an Optimized Condition-Based Maintenance System by Data Fusion and Reliability-Centered Maintenance." Reliability Engineering \& System Safety 95 (7): 786-96. doi:10.1016/j.ress.2010.02.016.

Penn State University, The Boeing Company, and MIMOSA. 2006. "Open Systems Architecture for Condition-Based Maintenance (OSA-CBM) - Primer.” MIMOSA.

Son, Jong-Duk, Gang Niu, Bo-Suk Yang, Don-Ha Hwang, and Dong-Sik Kang. 2009. "Development of Smart Sensors System for Machine Fault Diagnosis." Expert Systems with Applications 36 (9): 11981-91. doi:10.1016/j.eswa.2009.03.069.

Stauffer, J.-M., B. Dutoit, and B. Arbab. 2006. "Standard MEMS Sensor Technologies for Harsh Environment." In The Institution of Engineering and Technology Seminar on MEMS Sensors and Actuators, 2006, 91-96.

Trutt, F.C., J. Sottile, and J.L. Kohler. 2002. "Online Condition Monitoring of Induction Motors." IEEE Transactions on Industry Applications 38 (6): 1627-32. doi:10.1109/TIA.2002.804758. 
Wang, W., and O.A. Jianu. 2010. "A Smart Sensing Unit for Vibration Measurement and Monitoring." IEEE/ASME Transactions on Mechatronics 15 (1): 70-78. doi:10.1109/TMECH.2009.2016956.

Yun, Gun Jin, Soon-Gie Lee, Joan Carletta, and Tomonori Nagayama. 2011. "Decentralized Damage Identification Using Wavelet Signal Analysis Embedded on Wireless Smart Sensors." Engineering Structures 33 (7): 2162-72. doi:10.1016/j.engstruct.2011.03.007. 


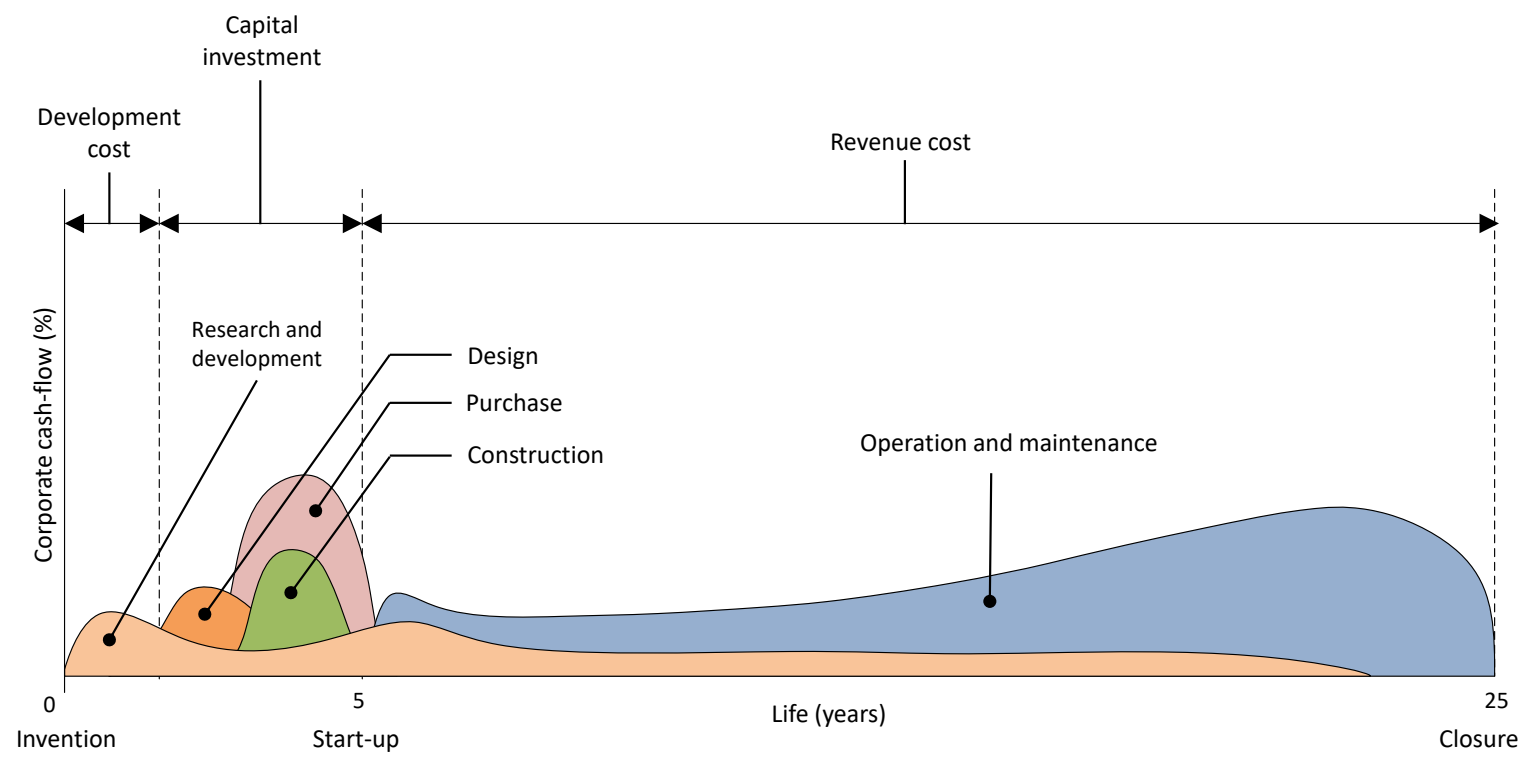

Figure 1: A lifecycle cost profile of a plant (Kelly 2006).

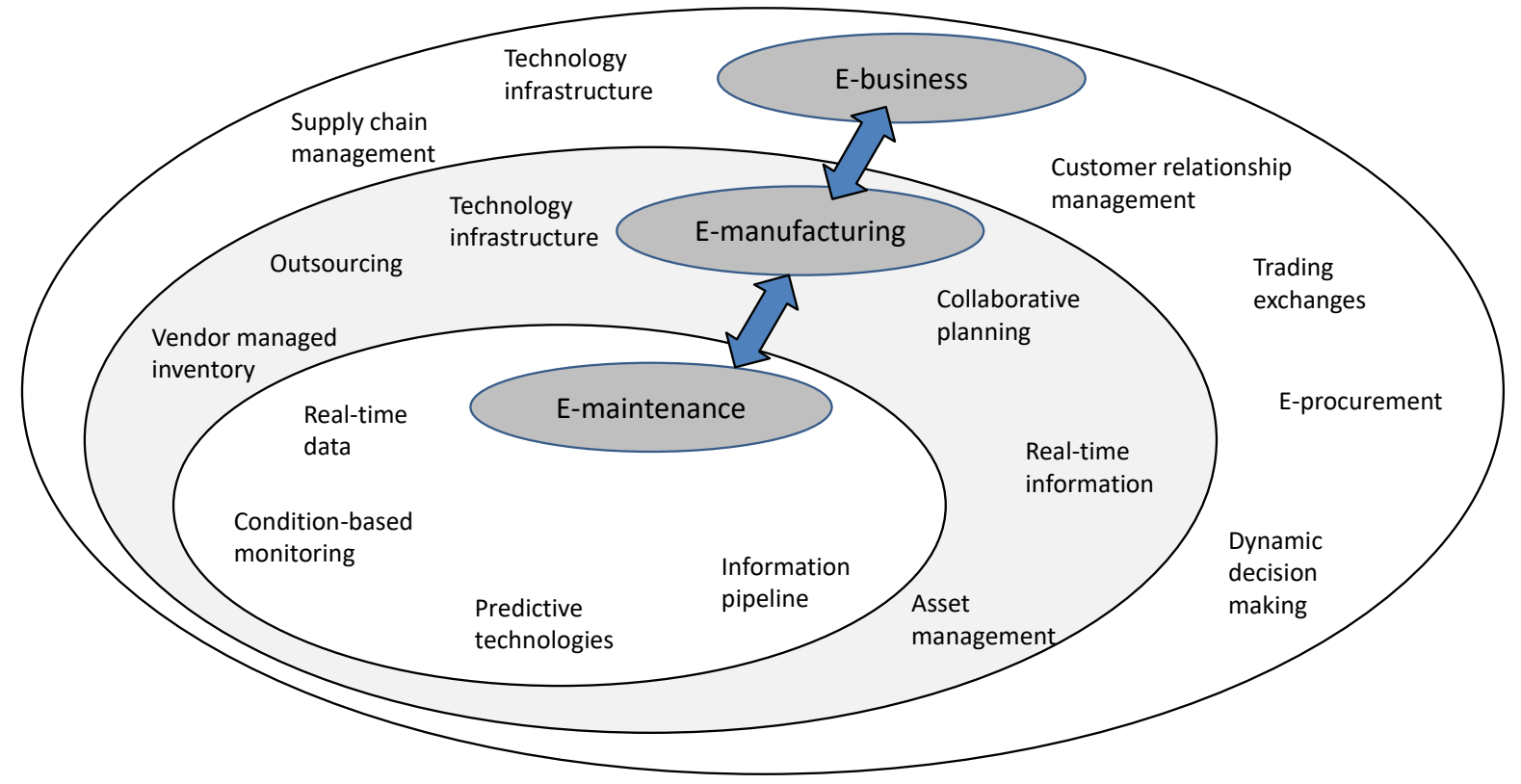

Figure 2: Integration of e-maintenance with e-manufacturing and e-business (Koc, Ni, and Lee 2002). 


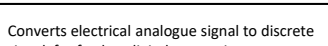

signal for further digital processing.

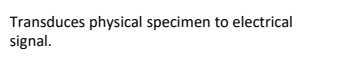

Processes input data using various signal processing techniques: filtering, $\mathrm{FFT}$, feature extraction, etc..

Gathers processed data and compare to specific pre-defined feature components

Assess machine health based on $\mathrm{CM}$ data and diagnostics records.

Conclude and predict future health of the assets based on certainty level and error

Generates report and make suggestion on .

Present information via man/machine interface.

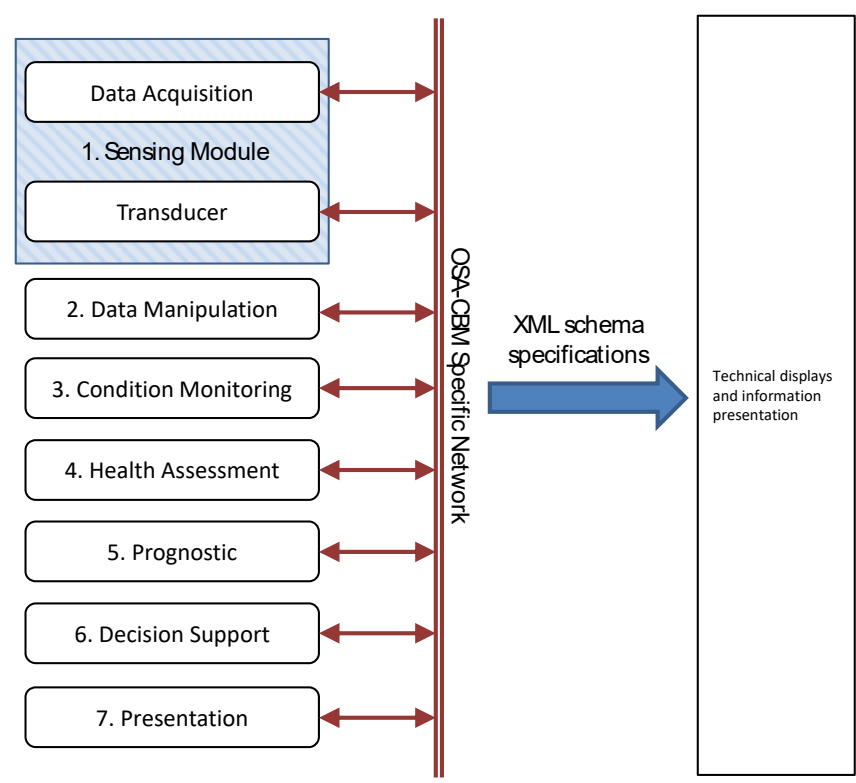

Figure 3: Open standard architecture of condition-based maintenance (Penn State University, The Boeing Company, and MIMOSA 2006).

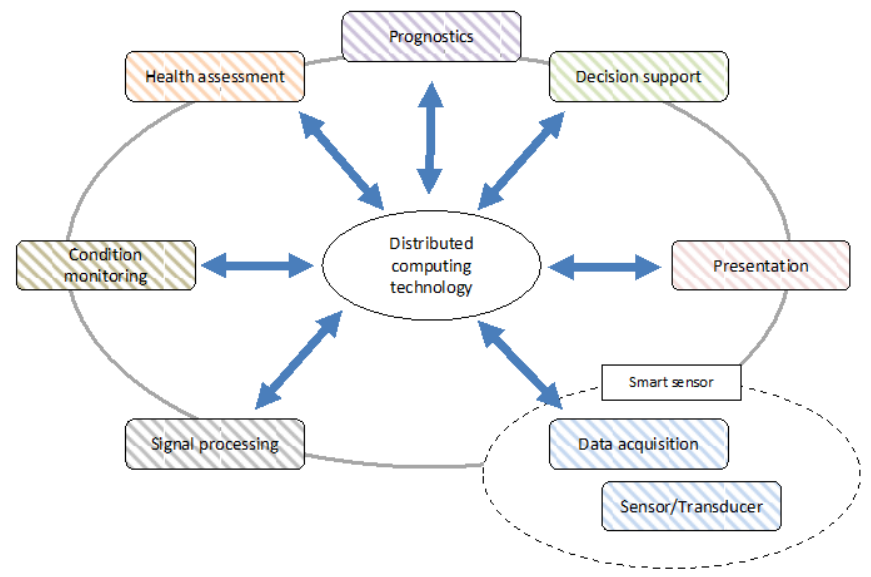

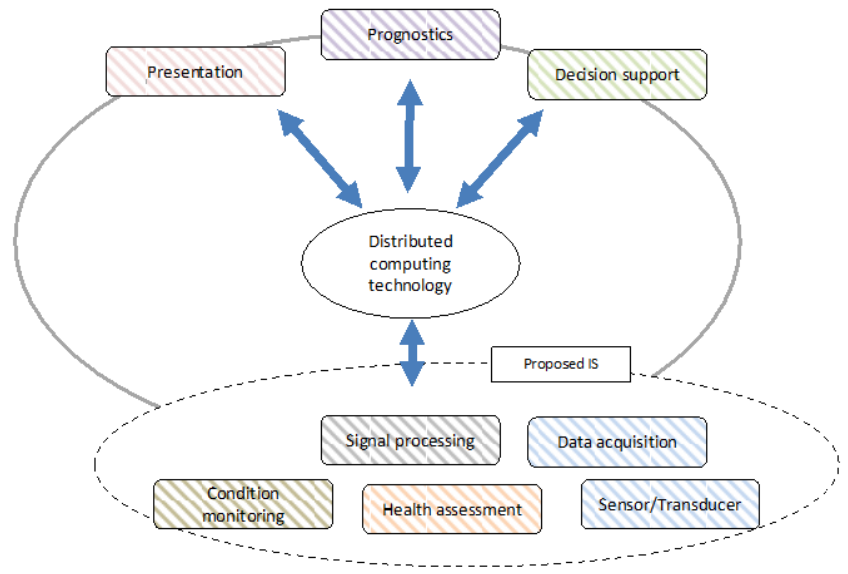

(b)

Figure 4 (a): The data circulation within an open system CBM design. All components are established based upon distributed computing topology; (b)The data circulation within a proposed IMSEM compatible with open system CBM design. A total of 5 functional blocks are implemented within a sensing module to reduce interface loads with other platforms. 


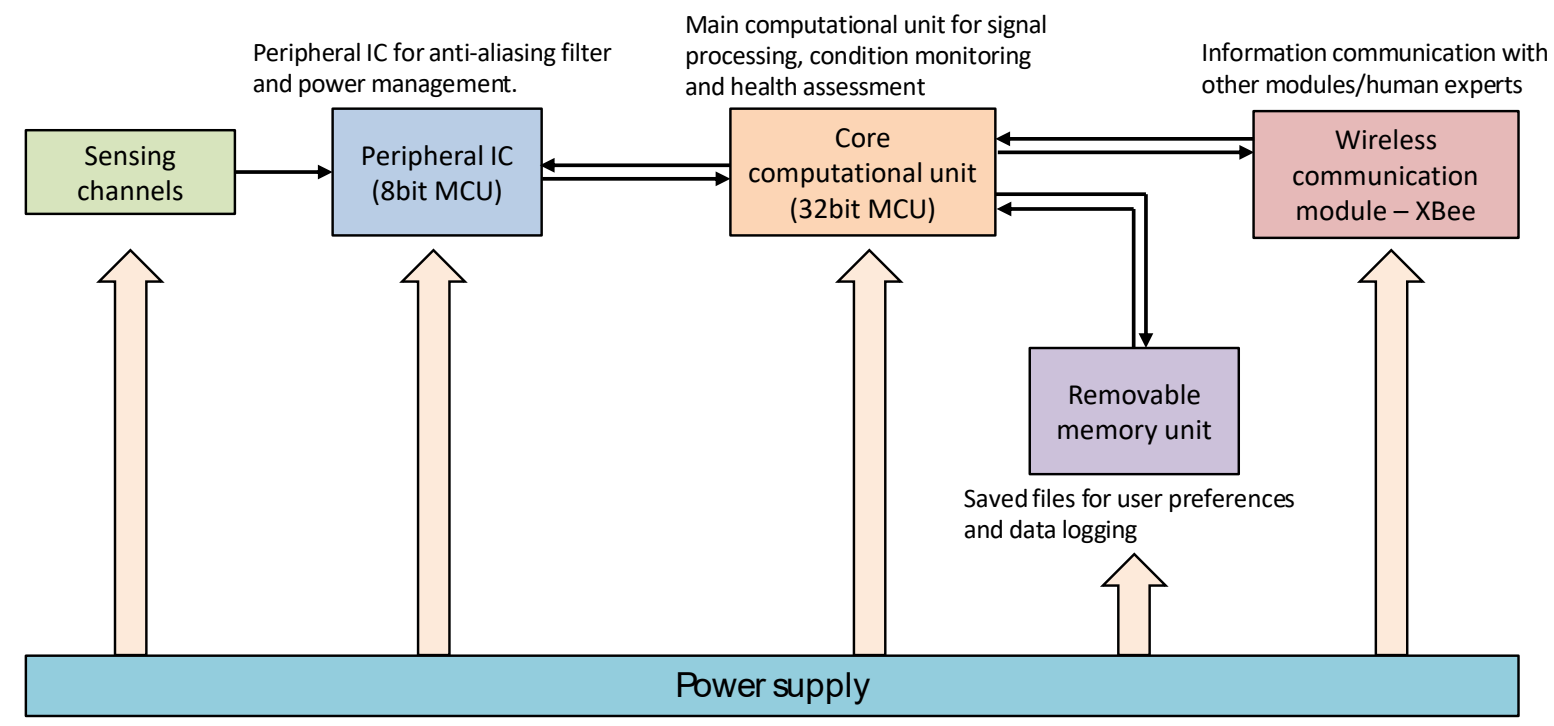

Figure 5: The structure of IMSEM for machinery condition-based maintenance.

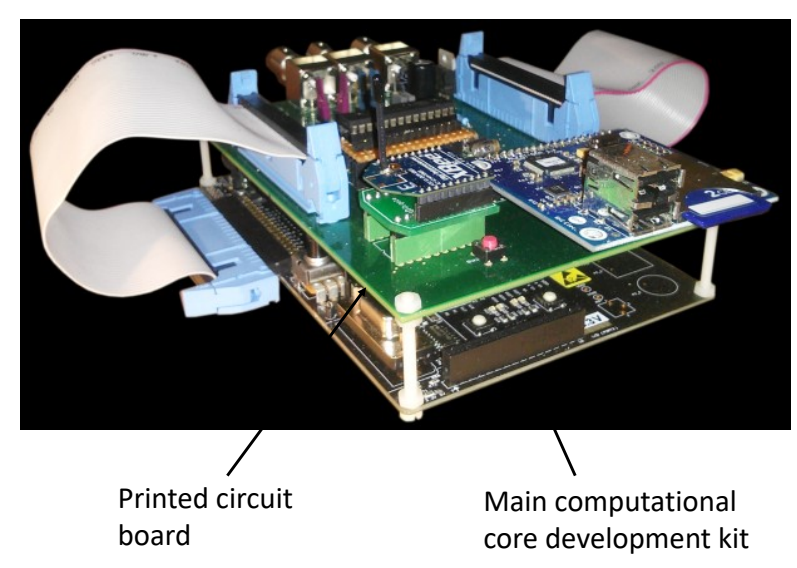

Perspective view

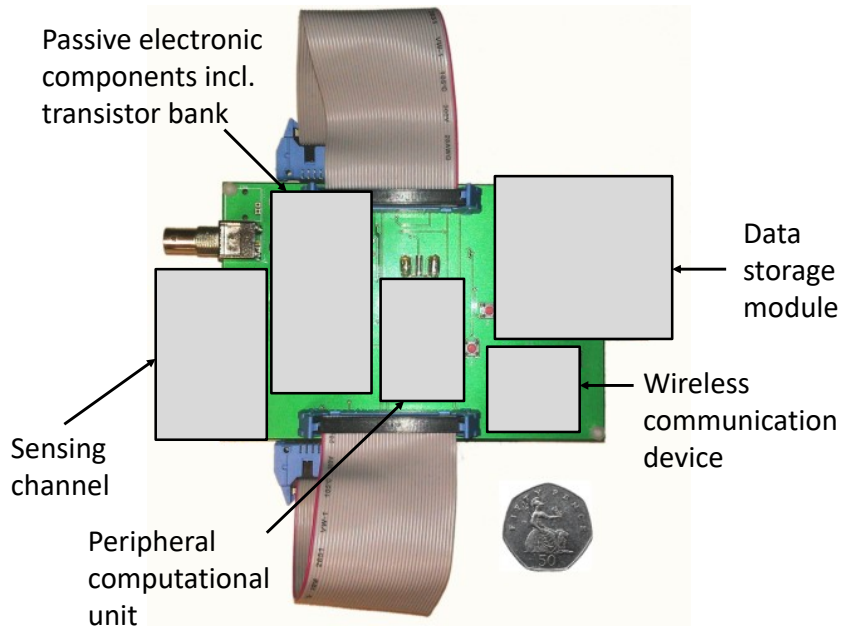

Top layout view

Figure 6: Working prototype of IMSEM.

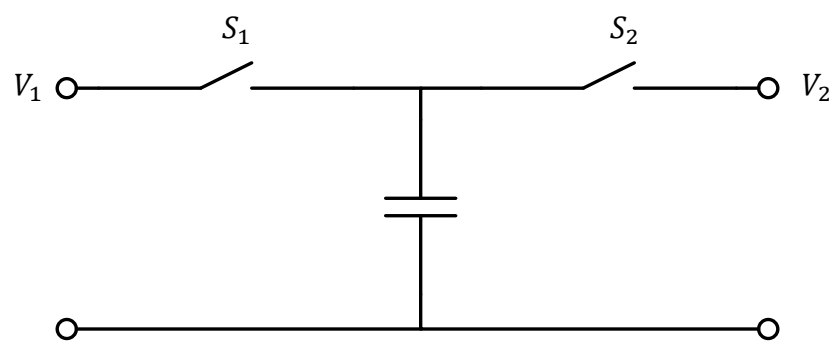

Figure 7: Simple switched capacitor circuit. 


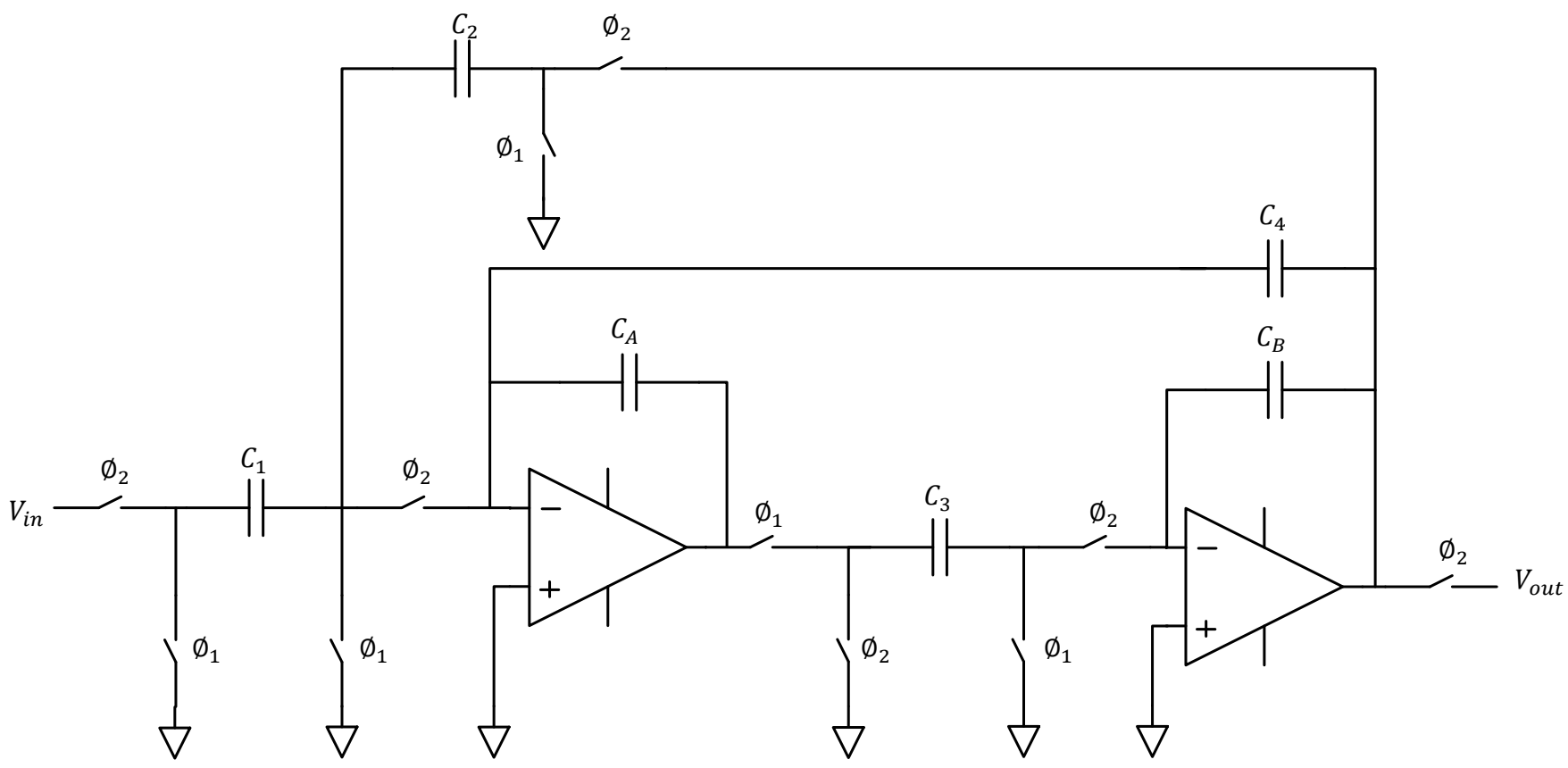

Figure 8: 2nd order state variable low-pass filter.
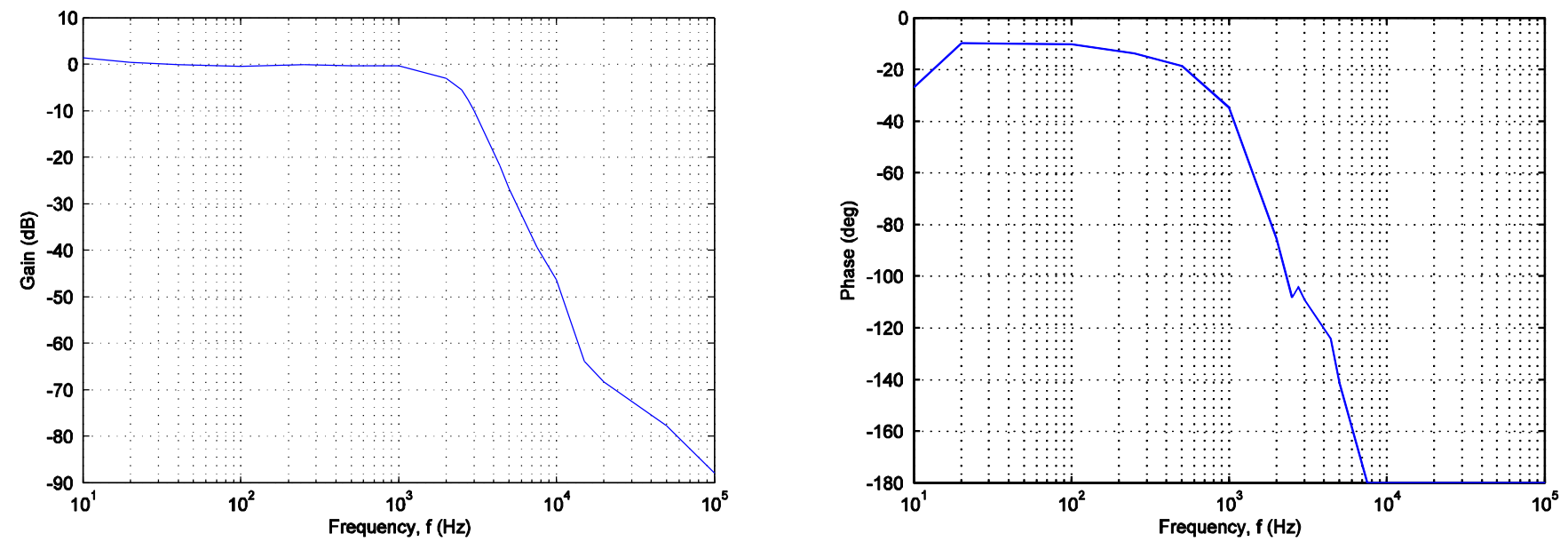

Figure 9: Frequency response of anti-aliasing with cut-off frequency of $2.5 \mathrm{kHz}$. 


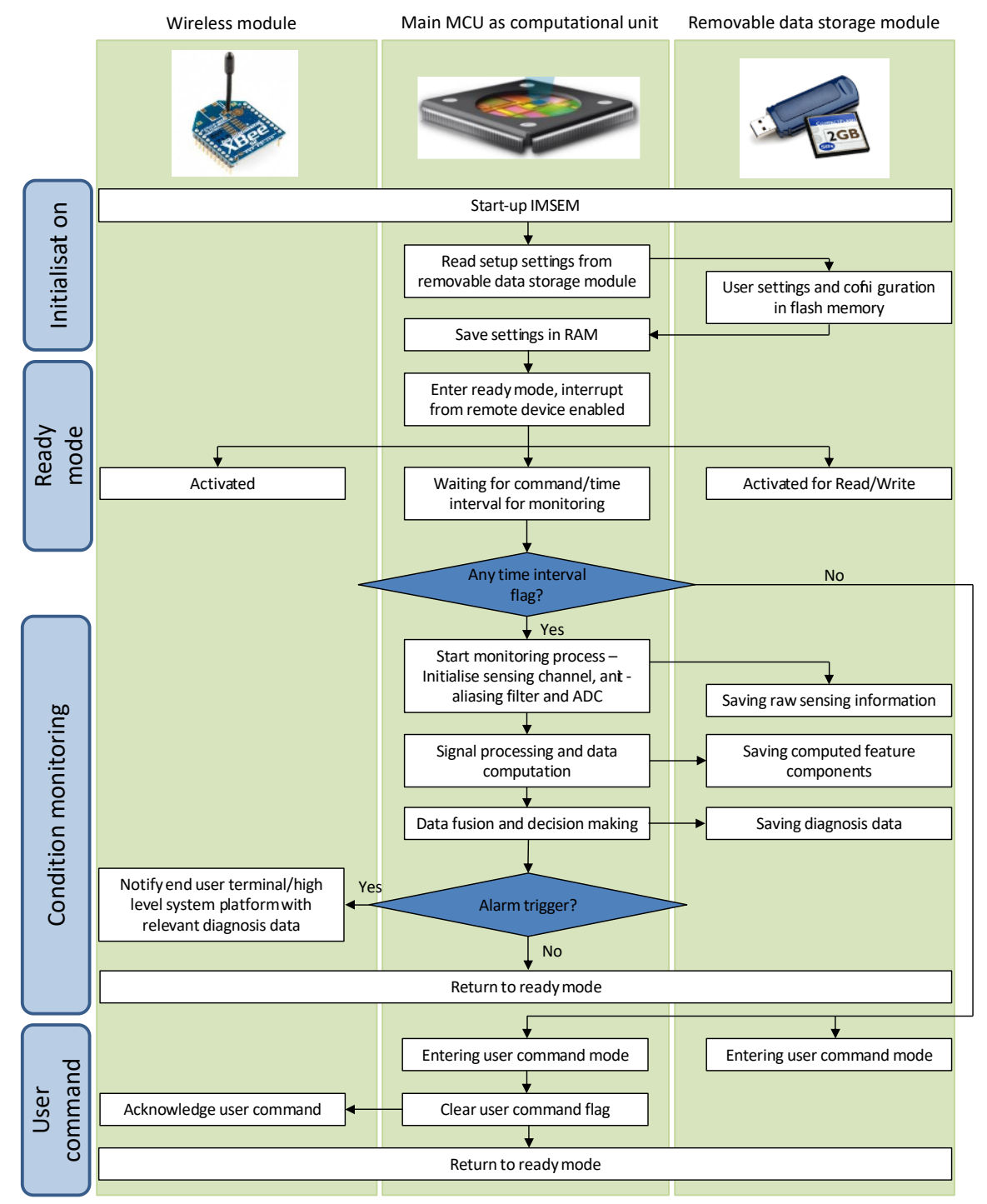

Figure 10: Data circulation within IMSEM. 


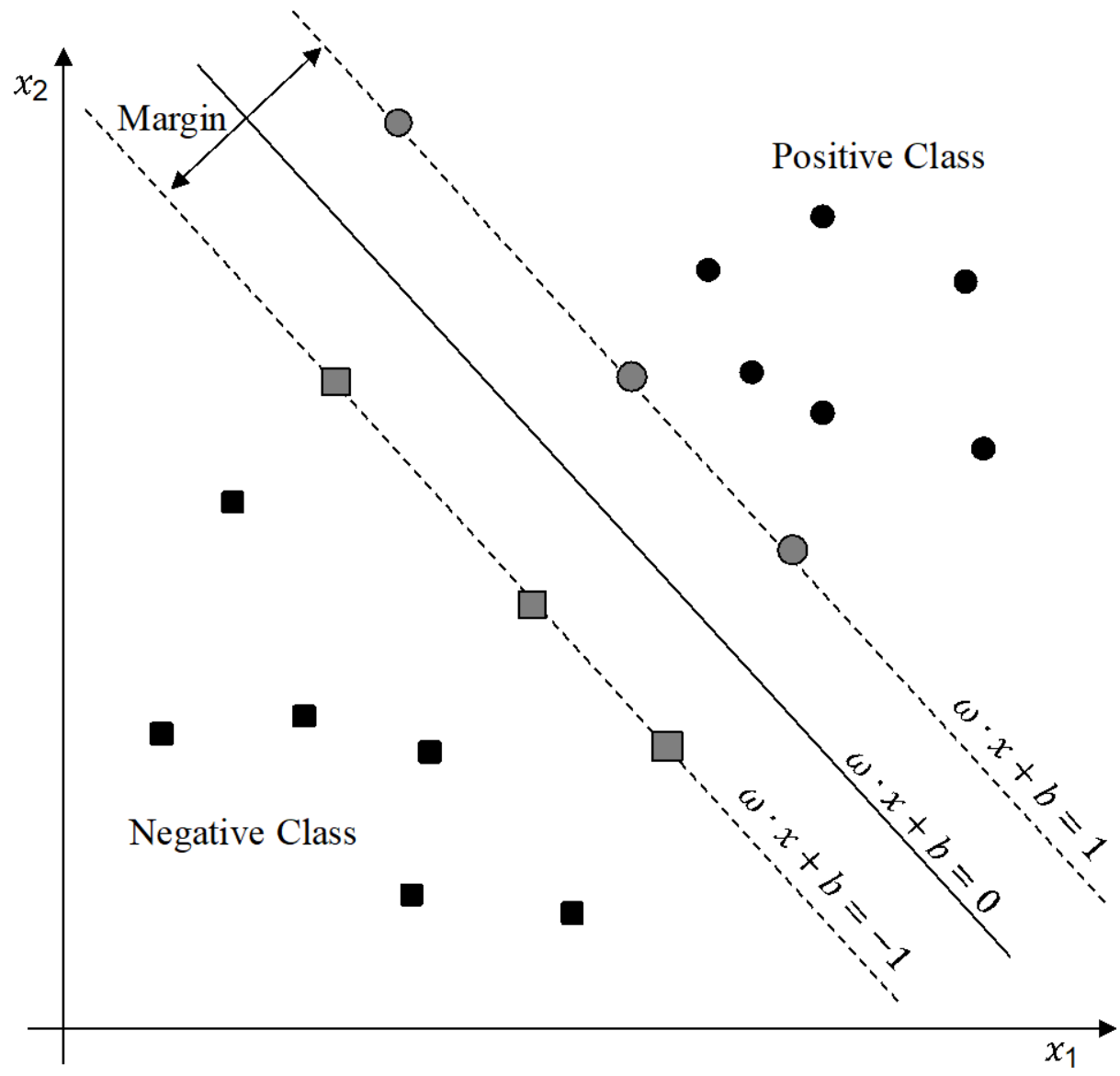

Figure 11: An example of 2-class classifier with two-dimensional feature vectors using SVM. 


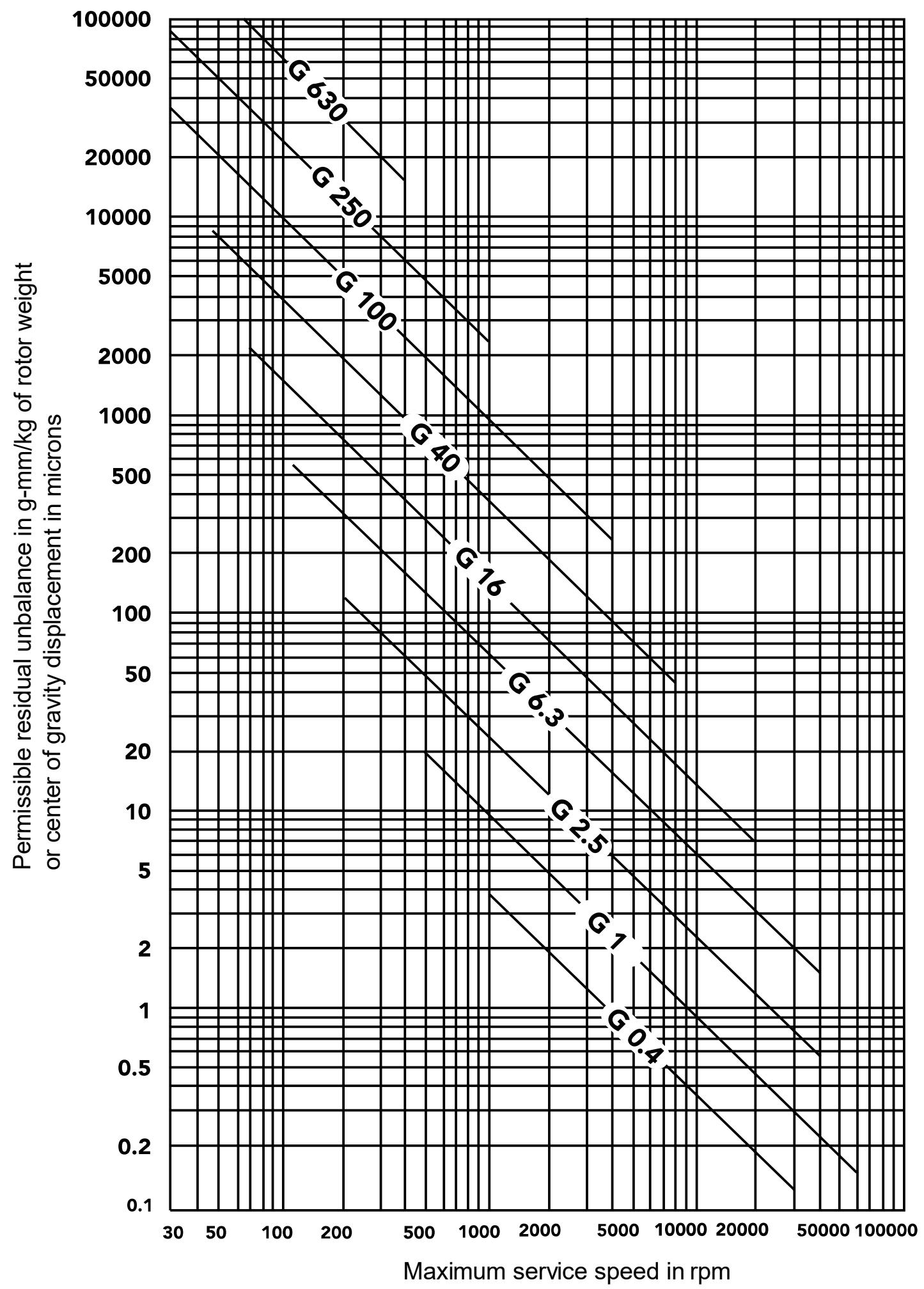

Figure 12: Maximum permissible residual unbalance, $e_{p}$ from ISO 1940-1:2003. 


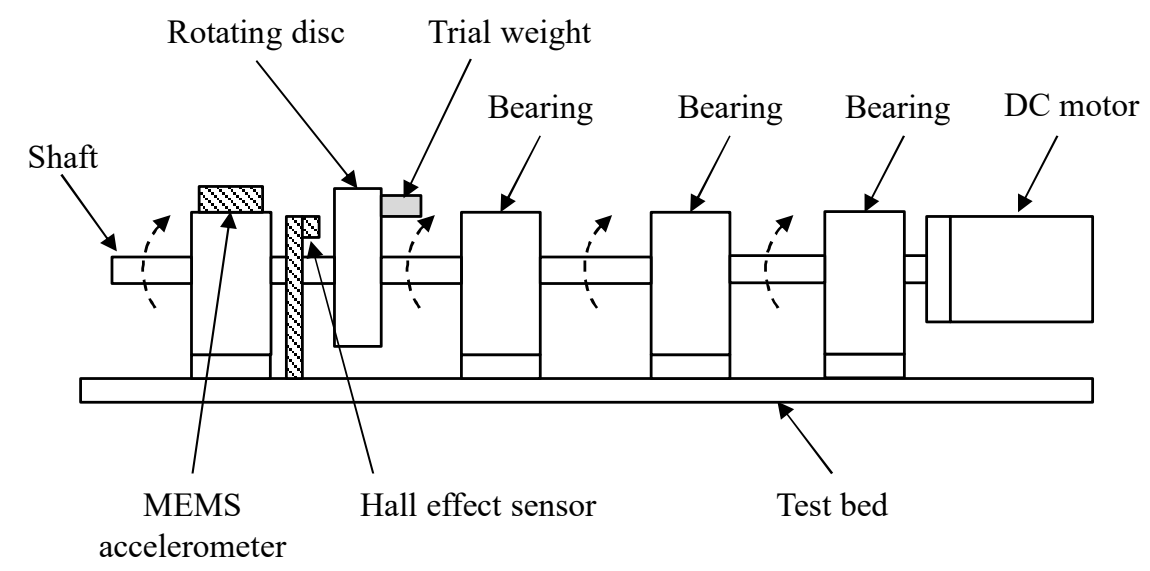

Figure 13: Outline of DC motor test rig with MEMS accelerometer and Hall effect sensor.

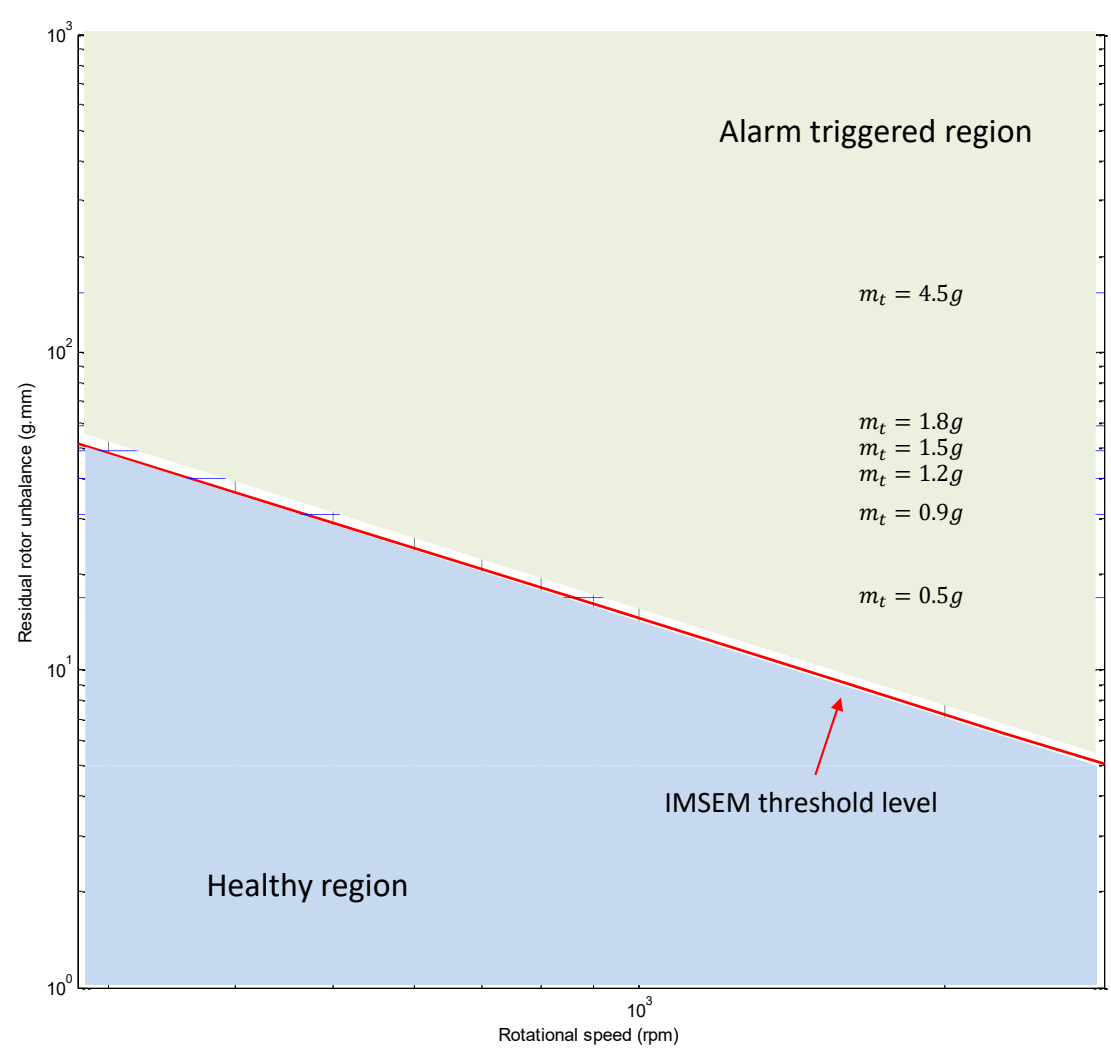

Figure 14: Rotor unbalance threshold over trial weights based on ISO 1940/1. The permissible threshold level decreases when rotational speed increases. 

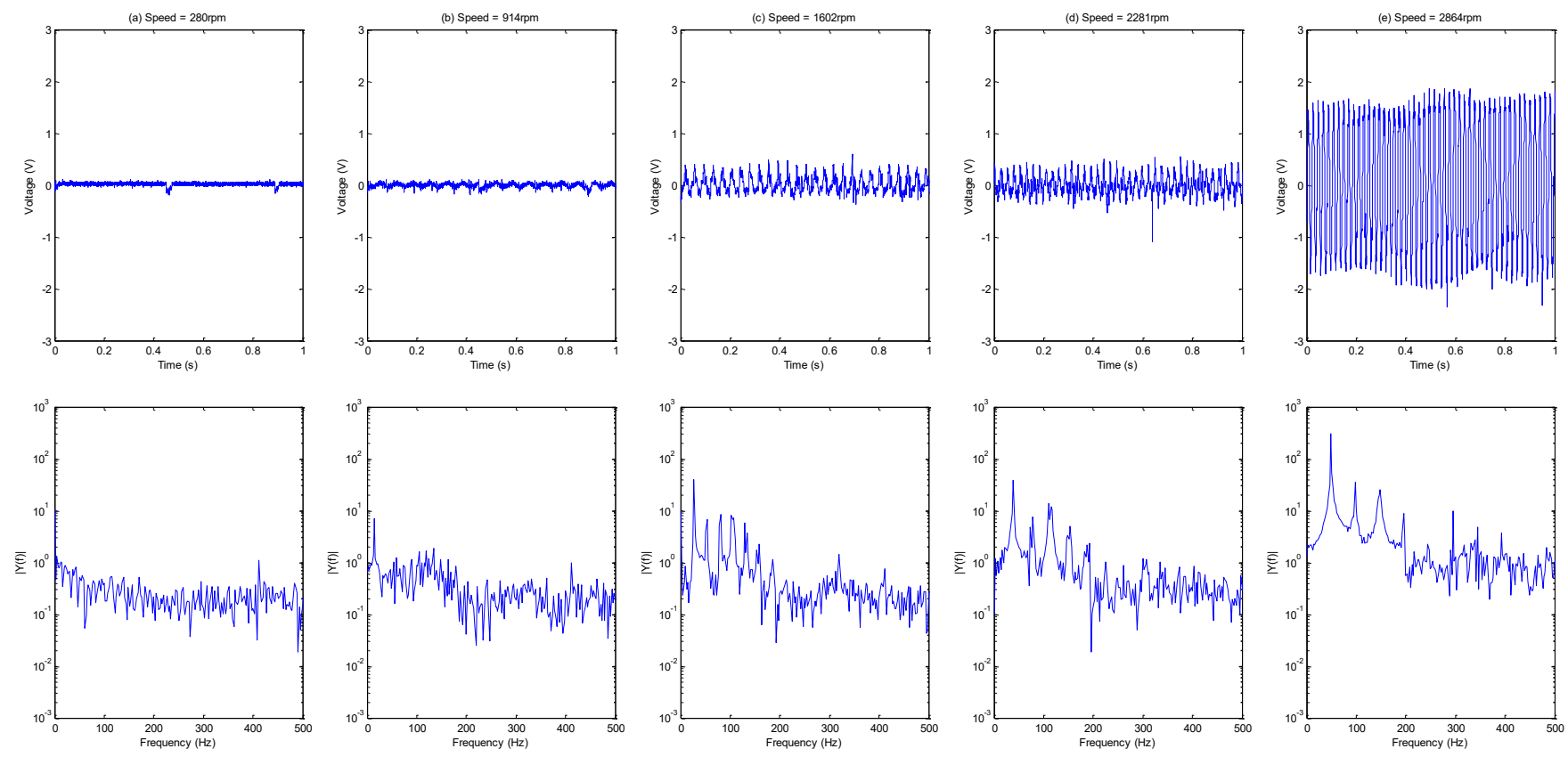

Figure 15: Vibration waveforms and power spectrum of acquired signals from IMSEM at different rotating speed: a) $280 \mathrm{rpm}$, b) $914 \mathrm{rpm}$, c) $1602 \mathrm{rpm}$, d) $2281 \mathrm{rpm}$ and e) $2864 \mathrm{rpm}$.
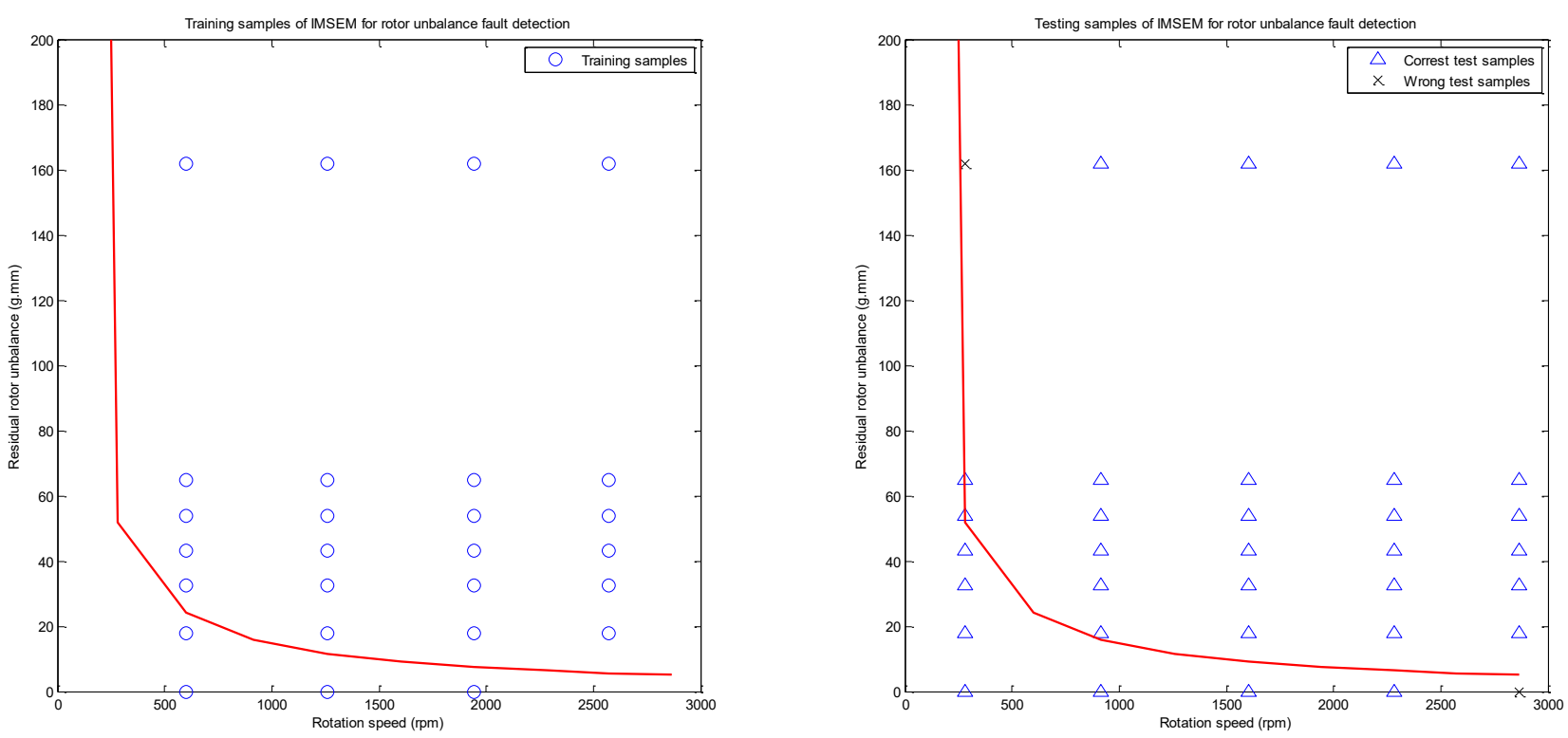

Figure 16: Mapping of training and test samples on ISO1940-1 guideline. 

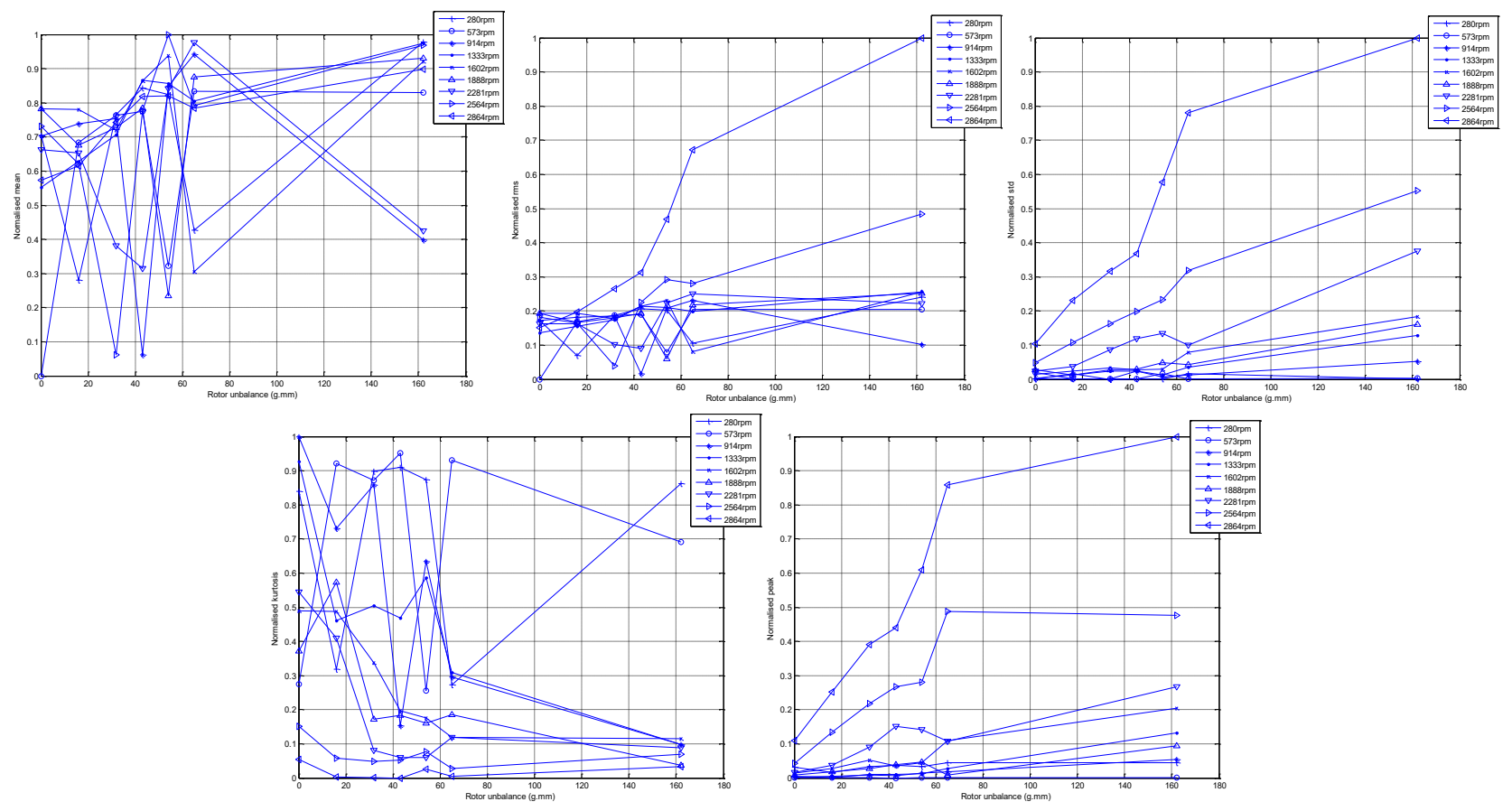

Figure 17 Feature components with different rotor unbalance and speed. 